Development of proliferative

\section{retinopathy in patients with long-standing insulin-dependent diabetes mellitus}

Abstract

Purpose and method Some patients with longstanding insulin-dependent diabetes mellitus (IDDM) develop little or no retinopathy. Using a clinic-based questionnaire and examination, we investigated a group of patients with over 40 years or more of IDDM who had been followed up at the King's Diabetes Centre for an average of 40 years. We compared those who had developed proliferative diabetic retinopathy during their disease with those with minimal or no retinopathy.

Results The study did not find any statistically significant differences between the two groups to suggest why some long-term insulin-dependent diabetics develop little retinopathy. Those who developed sightthreatening retinal complications did so at an average of 35 years after diagnosis and it resulted in little visual disablement. Very few patients in either group had developed other significant complications.

Conclusions This study details an interesting group of patients with long-term IDDM mellitus with a mean follow-up period of $\mathbf{4 0}$ years. Some patients with long-standing IDDM develop little or no retinopathy. With the advent of community ophthalmic screening, these patients are now rarely seen in the eye clinic. Those who did develop retinal complications and required treatment have remarkably little visual disablement. However, these complications developed late in the history of their disease, emphasising the need for continued screening.

Key words Insulin-dependent diabetes mellitus, Long term, Retinopathy

Insulin-dependent diabetes mellitus (IDDM) is a leading cause of legal blindness in people of working age. Duration of diabetes is a known risk factor for the development of retinopathy. ${ }^{1}$ It is unusual to find significant retinopathy
C.J. STYLES, W. DODDS, P. WATKINS,

D. MCHUGH, R. BLACH within 10 years of diagnosis but $95 \%$ will develop background retinopathy within 15 years and up to $70 \%$ develop proliferative retinopathy within 30 years. ${ }^{2,3}$ However, there are patients with long-standing IDDM with little or no retinopathy. The reasons for this are unclear, although the recent Diabetes Control and Complications Trial (DCCT) has indicated the importance of good control in slowing the progression of retinopathy. ${ }^{4}$ Other risk factors known to be important in the development of retinopathy include nephropathy and possibly hypertension., ${ }^{5,6}$ Pregnancy can also lead to a temporary worsening of retinopathy, perhaps in association with tight control. ${ }^{7}$

The aims of the study were to identify patients with IDDM of 40 years or more duration and mild, minimal or no retinopathy and compare them with patients with IDDM of a similar duration who developed proliferative retinopathy. The principal objective was to identify any characteristics which may account for the relative sparing of ocular complications in these patients.

\section{Materials and methods \\ Selection of patients}

Fifty-two patients were recruited from a database of patients from the King's Diabetes Centre at King's College Hospital, London. The majority of patients in this study had been seen at the centre since diagnosis and medical notes were available from this time.

The inclusion criteria were patients with IDDM for 40 years or more. IDDM was defined as occurring in those in whom the diagnosis was made before age 30 years and insulin therapy commenced at onset or within 1 year of diagnosis.

A questionnaire was completed by the patient, a research nurse and ophthalmologist. Participants provided information on social and medical history, details of their diabetes including past and present insulin therapy,
C.J. Styles

D. McHugh

R. Blach

Department of Ophthalmology King's College Hospital London, UK

W. Dodds

P. Watkins

King's Diabetes Centre King's College Hospital London, UK

Dr Caroline Styles Department of Ophthalmology Ninewells Hospital and Medical School Dundee DD1 9SY, UK

Tel: +44 (0)1382660111 Fax: +44 (0)1382 660130

Received: 18 January 2000 Accepted in revised form: 1 June 2000 
Table 1. Characteristics of the study population: I

\begin{tabular}{lllllc}
\hline Variable & Group 1 $(n=29)$ & Group 2 $(n=23)$ & d.f. & $t$ & $p$ \\
\hline Female & 22 & 15 & 50 & 0.83 & 0.41 \\
Age (years) & $63.8(53-76)$ & $59.6(45-77)$ & 50 & 2.02 & 0.02 \\
Age at diagnosis (years) & $14.4(2-29)$ & $14.1(3-26)$ & 50 & 0.14 & 0.89 \\
Duration of IDDM & $49.0(40-67)$ & $45.5(40-56)$ & 50 & 1.88 & 0.03 \\
Follow-up at King's College Hospital (years) & $45(25-61)$ & $40(14-55)$ & 50 & 2.31 & 0.03 \\
Body mass index & $25.8(17.2-38.4)$ & $26.0(20.8-33.8)$ & 45 & 0.17 & 0.43 \\
Height (m) & 1.64 & 1.57 & 45 & 0.46 & 0.32 \\
HbA $_{1 \mathrm{c}}(\%)$ & $8.3(5.8-12.3)$ & $8.7(6.7-11.9)$ & 50 & 1.00 & 0.16 \\
\hline
\end{tabular}

Results are given as mean with range in parentheses.

$p$ value calculated from Student's $t$-test.

hospital admissions specifically for diabetic

complications and treatment for retinal complications. In the majority of patients the medical notes were available to confirm the history given. Investigation included blood pressure measurement, urinalysis and blood was taken for creatinine levels, cholesterol and $\mathrm{HbA}_{1 \mathrm{c}}$ measurements. Results of urinalysis from two previous clinic visits were also recorded. The amount of albuminuria was quantified using the mean of at least two previous albumin:creatinine ratio measurements. All patients had an ocular examination consisting of visual acuity measurement, slit-lamp examination and fundoscopy following dilatation of the pupils.

The group was then divided into those with minimal, mild or no retinopathy (group 1) and those who had documented proliferative retinopathy either in the past or at present (group 2). Statistical analysis was then performed. The Mann-Whitney test was used to compare the two groups for non-parametric data. Parametric data were compared using Student's $t$-test. Two-tailed $p$ values are quoted.

\section{Definitions}

Minimal non-proliferative retinopathy was defined as fewer than three microaneurysms. Mild non-proliferative changes included microaneurysms, occasional intraretinal haemorrhage and hard exudates, and retinal oedema that did not encroach on the fovea.

Preproliferative retinopathy was defined as the presence of cotton wool spots, venous beading or loops and intraretinal microvascular abnormalities. Proliferative retinopathy was documented as the presence of neovascularisation either at the disc or elsewhere in the retina.

Microalbuminuria was defined as a negative Albustix measurement but an albumin-creatinine ratio of greater than $25 \mu \mathrm{g} / \mathrm{mmol}$ on at least two occasions. Proteinuria was defined as three consecutive positive Albustix measurements.

The number of cigarettes smoked was calculated as pack-years by multiplying the number of packs smoked per day (pack = 20 cigarettes) by the number of years over which that amount was smoked.

\section{Results}

The patients were divided into two groups depending on the retinal findings. Group 1 consists of 29 subjects. These patients had never had proliferative retinal complications. Group 2 consists of 23 subjects who had documented neovascularisation of the retina at some stage of their diabetes. The patients had been followed up at King's College Hospital for a mean of 45 years for group 1 and 40 years for group 2 (Table 1). Twenty-six patients (90\%) from group 1 and $18(78 \%)$ from group 2 have been seen in the department since their diagnosis. All the subjects were Caucasian. Fourteen (48\%) patients from group 1 and 12 (55\%) from group 2 had been smokers but only 3 patients in total still smoked. Tables 1 and 2 display the characteristics of the two groups obtained from the questionnaire and examination. Patients in group 1 who had not developed proliferative retinopathy were older $(p=0.02)$ and had a longer duration of IDDM $(p=0.03)$.

\section{Ocular features}

At the time of examination 14 patients in the untreated group 1 had minimal or no retinopathy. The other 15 had mild retinopathy. In group 2, 9 patients had retinal neovascularisation, 12 had mild retinopathy and in 2 there was no view of the fundus due to cataract. Both of these last patients had documented neovascularisation in the past.

The visual acuity in both groups was relatively well preserved. Twenty-seven patients (93\%) in group 1 and $16(70 \%)$ in group 2 had a visual acuity of at least 6/9 in one eye. Three patients were registered blind and 2 partially sighted. This was due to diabetes in 4 cases, all

Table 2. Characteristics of the study population: II

\begin{tabular}{|c|c|c|c|c|}
\hline Variable & $\begin{array}{l}\text { Group } 1 \\
(n=29)\end{array}$ & $\begin{array}{l}\text { Group } 2 \\
(n=23)\end{array}$ & $z$ & $p$ \\
\hline $\begin{array}{l}\text { Alcohol intake } \\
\text { (units/week) }\end{array}$ & $3.0(0-16)$ & $3.0(0-14)$ & 1.01 & 0.31 \\
\hline Smoking (pack-years) & $0(0-36)$ & $6.2(0-60)$ & 0.50 & 0.62 \\
\hline $\begin{array}{l}\text { Hospital admissions for } \\
\text { diabetic complications }\end{array}$ & $1(0-12)$ & $1(0-60)$ & 0.25 & 0.81 \\
\hline Creatinine $(\mu \mathrm{mol} / \mathrm{l})$ & $78(56-330)$ & $89(63-432)$ & 1.50 & 0.15 \\
\hline
\end{tabular}

Results given as median with range in parentheses. $p$ value calculated using the Mann-Whitney test.

Abnormal creatinine values for group $1=330$, group $2=205$, 348,432 . 
Table 3. Reasons for laser treatment

\begin{tabular}{lcc}
\hline Reason & $\begin{array}{c}\text { No. of patients } \\
(n=22)\end{array}$ & \% of group \\
\hline NVD & 10 & 45 \\
NVE & 2 & 9 \\
NVD and NVE & 2 & 9 \\
NV unspecified & 5 & 23 \\
NV and maculopathy & 3 & 14 \\
\hline
\end{tabular}

NVD, new vessels at the optic disc; NVE, new vessels elsewhere in the retina; $\mathrm{NV}$, new vessels.

of whom were in group 2. The fifth case was due to glaucoma and this patient had no diabetic retinopathy. Nine (31\%) patients from group 1 and 5 (22\%) from group 2 had undergone cataract extraction at a mean age of 59 years (range $44-74$ years).

No patients in group 1 had had laser treatment. Twenty-two of 23 of the patients in group 2 had had laser treatment. One patient in this group had developed neovascularisation in 1965 and had no treatment. She subsequently developed rubeotic glaucoma. The reasons for laser treatment are shown in Table 3. The laser treatment was carried out at a median of 35 years (range 15-50 years) after diagnosis of diabetes. Only one patient had laser treatment in the 1970s when it first became available at King's. The remaining patients were treated between 1980 and 1996. Three patients had laser to the macula as well as panretinal photocoagulation for neovascularisation. In all 3 patients this was for a localised area of macular oedema and was carried out at the same time as the panretinal photocoagulation.

\section{Diabetes}

There was no significant difference between groups in the type of insulin that the patients were taking. All patients had originally used porcine- or bovine-derived insulin and had changed to a human insulin at some point during the course of their diabetes. Twenty-six patients $(90 \%)$ from group 1 had a twice-daily regimen using two different types of insulin, compared with $13(57 \%)$ in group $2(p=0.006)$. Ten patients $(43 \%)$ in group 2 had a three or four times daily insulin regimen. The number of hospital admissions due to diabetes was low for both groups. Eight (28\%) patients in group 1 and $4(17 \%)$ in group 2 had no admissions.

Nine (31\%) patients from group 1 and 11 from group 2 $(48 \%)$ were taking hypotensive agents $(p=0.22)$, including 4 patients from group 1 and 9 from group 2 taking angiotensin converting emyzme inhibitors. There was no significant difference in mean diastolic or systolic blood pressures between the two groups. No patients were on lipid-lowering therapy but 4 commenced treatment following investigation undertaken as part of this study. There was no significant difference in any of the biochemical tests undertaken as part of the study.

\section{Nephropathy}

Twenty-five patients in group $1(86 \%)$ and 18 in group 2 (78\%) had three negative Albustix measurements and all these patients had a normal serum creatinine. Four (14\%) of these patients from group 1 and 3 (13\%) from group 2 had microalbuminuria determined from the measured albumin:creatinine ratio. Of those patients with positive Albustix measurements, 3 were in renal failure.

\section{Discussion}

The most striking feature of this group of long-term survivors with IDDM is the rarity of disabling retinal complications. Even in those who developed diabetic retinopathy requiring treatment the sight is relatively well preserved. Only 4 patients have visual impairment due to diabetes sufficient to qualify for blind or partial sight registration. This group of patients is, however, a 'survivor' group, and those with more serious retinopathy, blindness and other complications of diabetes may have died earlier in the course of their dis ase. In those patients who did develop proliferative diabetic retinopathy, treatment was initiated at a median of 35 years after diagnosis. Seventy-five per cent of group 2 commenced treatment more than 30 years after diagnosis, thereby representing a group with late-onset complications and emphasising the need for continued retinal screening even in these long-term patients.

We examined in more detail those factors known to be associated with worsening of diabetic retinopathy. The importance of good blood glucose control in long-term IDDM patients without retinopathy has been evaluated over a 9 year follow-up period. ${ }^{8}$ Despite medical notes being available in the majority of patients in our study, it is difficult to chart the blood glucose control over more than 40 years because for most of this period there have been no accurate tests reflecting long-term control.

Ninety per cent of the patients in group 1 with minimal or no retinal complications were on a twicedaily regimen of insulin compared with $57 \%$ of group 2 $(p=0.006)$. This conventional regimen was shown in the $\mathrm{DCCT}^{4}$ to be less effective than more intensive insulin regimens in delaying the onset and slowing progression of diabetic retinopathy. Our results may reflect an attempt to improve glycaemic control following the development of retinal complications in group 2 .

Nephropathy has been found to be important in the development of retinal complications and is also one of the major determinants of the prognosis of patients with IDDM. ${ }^{5,9}$ The majority of diabetics in our study, especially those without serious retinopathy (group 1), were spared this complication. However, patients with serious nephropathy would have died at an earlier stage of their disease and are therefore selected out of our survivor cohort.

Long-term survivors with IDDM of more than 40 years' duration can be entirely free from retinopathy or show any minimal changes which have not progressed over many years. Proliferative changes had occurred in 
approximately half of our patients and they required treatment relatively late in the course of their disease, but overall suffer little visual disability. No significant demographic differences were found between those who did and did not develop serious retinopathy. However, this group of diabetic patients emphasises that continuing surveillance is required to detect treatable retinopathy even after several decades of IDDM.

We thank David Chan and Richard Hooper for their help with statistical analysis.

\section{References}

1. West K, Erdrich L, Stober J. A detailed study of risk factors for retinopathy and nephropathy in diabetes. Diabetes 1980;29:501-7.

2. Klein R, Klein BEK, Moss SE, Davis MD, DeMets DL. The Wisconsin Epidemiological Study of Diabetic Retinopathy II. Prevalence and risk of diabetic retinopathy when age at diagnosis is less than 30 years. Arch Ophthalmol 1984;102:520-6.
3. Orchard T, Dorman J, Maser R, Becker D, Drash A, Ellis D, et al. Prevalence of complications in IDDM by sex and duration. Pittsburgh Epidemiology of Diabetes Complications study II. Diabetes 1990;39:1116-24.

4. DCCT Research Group. The effect of intensive treatment of diabetes on the development and progression of long term complications in insulin dependent diabetes. N Engl J Med 1993;329:977-1034.

5. Lloyd C, Orchard T. Diabetes complications: the renal retinal link. An epidemiological perspective. Diabetes Care 1995;18:1034-8.

6. Borch-Johnsen K, Nissen H, Henriksen E, Kreiner S, et al. The natural history of insulin-dependent diabetes mellitus. 1. Long term survival with and without late diabetic complications. Diabetic Med 1987;4:201-10.

7. Klein B, Moss SE, Klein R. Effect of pregnancy on the progression of diabetic retinopathy. Diabetes Care 1990;13:34.

8. Kullberg C, Arnquist H. Good blood glucose control characterises patients without retinopathy after long diabetes duration. Diabetic Med 1995;12:314-20.

9. Chavers BM, Mauer SM, Ramsey RC, Stettes MW. Relationship between retinal and glomerular lesions in IDDM patients. Diabetes 1994;43:441-6. 